\title{
Lyrisme de la vanité et vanité du lyrisme. Quelques exemples de poétiques du XIX' siècle, de Baudelaire à Laforgue
}

Si l'on se réfère à la tradition, la Vanité peut être définie comme un tableau visuel ou textuel symbolisant l'inanité de la condition humaine et incitant le spectateur / lecteur au mépris du monde terrestre. Articulant représentation symbolique et discours moral, la Vanité, picturale ou littéraire, atteint son apogée au XVII ${ }^{e}$ siècle (Lanini, 2006). Grâce à sa codification, qui l'érige en genre à part entière, elle perdure au-delà de cette époque propice à son épanouissement. Delphine Gleizes (2002/2004, p. 75-91) a notamment montré comment on la retrouvait dans la littérature romanesque du XIXe siècle. Sans prétendre à l'exhaustivité, nous nous proposons d'interroger ces réminiscences en analysant quelques exemples dans la poésie lyrique de la seconde moitié du siècle. De même que la dimension religieuse et dogmatique tend à s'estomper au XIX', les symboles de la vacuité et de l'illusion existentielle sont repris mais de manière partielle. Peut-on alors encore parler de Vanité quand le discours symbolique est lacunaire ou incomplet ? Notre hypothèse est que sa déconstruction participe au renouvellement du lyrisme : la Vanité devient un instrument critique pour interroger la représentation du sujet et de ses émotions.

\footnotetext{
- Sandra Glatigny - professeur agrégée des Lettres modernes au Lycée Pierre-Corneille de Rouen, docteur en littérature comparée, chercheur associé au CÉRÉdI (Centre d'études et de recherches éditer-interpréter) de l'Université de Rouen. Adresse pour correspondance : 68 C, rampe Bouvreuil, 76000 Rouen, France; e-mail : sandra.glatigny@gmail.com
} 


\section{Modèles et transpositions}

La reprise de la Vanité consiste d'abord dans la transposition littéraire de l'iconographie : imagerie macabre, portraits, natures mortes mettent en scène le caractère éphémère de l'existence humaine et la vacuité des divertissements auxquels l'homme se livre pour l'oublier. Dans Les Fleurs du Mal de Baudelaire, de nombreux titres font référence à l'ars moriendi médiéval : "L'Amour et le Crâne " (1980, p. 88-89), «Le Squelette laboureur» (1980, p. 69-70) ou «Danse macabre» (1980, p. 71-73), "Le Revenant » (1980, p. 47-48), "Le Mort joyeux » (1980, p. 51-52). L'image du squelette et du crâne est au cœur de cette évocation :

Ses yeux profonds sont faits de vide et de ténèbres,

Et son crâne, de fleurs artistement coiffé,

Oscille mollement sur ses frêles vertèbres.

Ô charme d'un néant follement attifé. (Baudelaire, 1980, p. 72)

Baudelaire joue sur le contraste entre rire et sentiment de désespoir tragique pour signifier à l'homme son impuissance à lutter contre le temps. Dans « Une charogne ", l'évocation réaliste et macabre de la décomposition participe de la stratégie qui consiste à terrifier le lecteur en lui présentant concrètement l'image de son devenir ou de son anéantissement. La conclusion est implacable :

- Et pourtant vous serez semblable à cette ordure,

À cette horrible infection, (Baudelaire, 1980, p. 23-24)

Ce poème, qui n'est pas sans évoquer le sonnet du poète de la Renaissance, Chassignet, «Mortel, pense quel est dessous la couverture (1967, p. 159-160), rappelle les effets physiologiques et chimiques de la mort sur le corps humain pour signifier l'inanité de l'existence humaine. Parmi les vers hérités de l'imagerie macabre, "La Morgue », dans Névroses de Maurice Rollinat, offre une description du lieu et, par conséquent, de ses hôtes :

Visages gonflés et difformes ;

Crânes aplatis ou fendus ;

Torses criblés, ventres énormes,

Cous tranchés et membres tordus :

Ils reposent comme des masses,

Trop putréfiés pour Clamart,

Ébauchant toutes les grimaces

De l'enfer et du cauchemar. (1885, p. 374) 
Le sujet constitue la médiation à l'origine de la vision funèbre. L'indétermination et la pluralité de l'évocation confèrent l'universalité au propos qui s'articule autour de la dimension esthétique des cadavres : le lecteur est confronté à une projection de son apparence une fois mort. Plus éthérée, l'écriture de Laforgue se nourrit en outre de références intertextuelles à la tradition moraliste :

Vermis sum, pulvis es! où sont mes nerfs d'hier?

Mes muscles de demain ? Et le terreau si fier

De Mon âme, où donc était-il, il y a mille

Siècles ! Et comme, incessamment, il file, file !...

Anonyme! Et pour Quoi ? - Pardon, quelconque Loi !

L'être est forme, Brahma seul est Tout-Un en soi. (1981, p. 58)

Le poète reprend les termes du psaume XXI attribué à David et un discours qui, même s'il ne s'inscrit pas dans la perspective judéo-chrétienne, rejoint la logique morale de la Vanité classique : l'existence terrestre est dérisoire, seule l'existence céleste est souhaitable. Cette reprise est l'occasion d'une plainte élégiaque du sujet.

Pourtant habitué du dialogue intersémiotique, Verlaine s'inspire peu de l'iconographie des Vanités. Peu de poèmes sont consacrés directement à la mort en tant que phénomène physique. Le poète préfère une vision allégorique comme celle du cavalier qui, à l'instar de la faucheuse dans « Mors » d'Hugo (1972, p. 280), présente les traits effrayants de la fin. Verlaine emprunte «Ce cavalier/ Des ballades d'Allemagne » qui :

- Tel l'ouragan sur la grève, -

D'une main tenant un glaive

Et de l'autre un sablier (1962, p. 66)

vient prendre ces victimes. La légende du Erlkönig, actualisée par Goethe, offre une représentation fantastique de l'angoisse de la mort, vue par l'énonciateur.

La Vanité textuelle s'accompagne d'un discours moralisateur qui rappelle l'imminence de la fin comme dans « Memento quia pulvis es", poème liminaire du recueil de Rollinat :

Crachant au monde qu'il effleure

Sa bourdonnante vanité,

L'homme est un moucheron d'une heure

Qui veut pomper l'éternité.

C'est un corps jouisseur qui souffre,

Un esprit ailé qui se tord :

C'est le brin d'herbe au bord du gouffre,

Avant la Mort. [...] 
Enfin, l'homme se décompose,

Sémiette et se consume tout.

Le vent déterre cette chose

Et l'éparpille on ne sait où.

Et le dérisoire fantôme,

L'oubli vient, s'accroupit et dort

Sur cette mémoire d'atome,

Après la Mort ! (1885, p. VII-VIII)

L'isotopie aérienne et la comparaison entre l'homme et l'insecte mettent l'accent sur le caractère dérisoire de l'homme. L'efficacité du propos résulte également de la versification : le pentasyllabe en fin de strophe vient signifier la brièveté de la vie. Dans Les Complaintes, Laforgue déplore également la volonté de l'homme de détourner son destin :

L'Homme et sa compagne sont serfs

De corps, tourbillonnants cloaques

Aux mailles de harpes de nerfs

Serves de tout et que détraque

Un fier répertoire d'attaques. (1981, p. 124)

La vision péjorative du corps humain est accentuée par les sonorités dysphoniques générées par les rimes en palato-vélaires. En outre, le poème développe le thème du divertissement pascalien :

Propre et correct en ses ressorts,

S'assaisonnant de modes vaines,

Il s'admire, ce brave corps,

Et s'endimanche pour sa peine,

Quand il a bien sué la semaine. [...]

Il se soutient de mets pleins d'art,

Se drogue, se tond, se parfume,

Se truffe tant, qu'il meurt trop tard ;

Et la cuisine se résume

En mille infections posthumes. (Laforgue, 1981, p. 124)

L'accumulation de verbes d'actions renvoie non seulement à l'activité désordonnée de l'homme qui cherche à s'étourdir pour oublier son néant, tel que le dépeint Pascal, mais également à la prolifération d'objets dans les Vanités picturales. Que l'on pense à L'Autoportrait avec symboles de vanités de David Bailly, peint en 1651 : bijoux, fleurs, instruments de musique, pipe, verre de vin, pièces de monnaie forment un en- 
semble apparemment hétéroclite, signifiant la dispersion et la vanité de l'existence humaine. Cet effet de prolifération est flagrant dans la " Grande complainte de Paris » (Laforgue, 1981, p. 152-154) qui dénonce la survalorisation des biens matériels, rejoignant ainsi l'héritage de la Nature morte édifiante. Cependant, au tableau figé, Laforgue substitue la dynamique du bonimenteur. Parmi les formes reprises se trouvent ce que l'on pourrait qualifier avec Edwige Keller-Rahbé de « récit-vanité » (2005, p. 246), c'est-à-dire un portrait en actes exemplaire, mettant en scène le renoncement aux divertissements vains. Corbière recourt fréquemment à cette forme, même si la portée en est souvent détournée. "Épitaphe » raconte l'histoire du poète maudit retiré et seul et se conclut par ces vers :

Il mourut en s'attendant vivre

Et vécut, s'attendant mourir. (Corbière, 2018, p. 74)

Le parallélisme et le chiasme entre les deux vers mettent en évidence le refus de renoncer aux activités terrestres. Corbière relate l'existence d'un poète qui ne maitrise pas la «bonne mort». Le récit fonctionne comme un contre-exemple.

On peut donc constituer un corpus de textes qui s'inscrivent dans la tradition de la Vanité dans la poésie de la deuxième moitié du XIX ${ }^{e}$ siècle. Cependant, on remarque que la configuration symbolique et morale est mise en péril par le caractère parcellaire de la reprise.

\section{Décomposition}

Si elles reprennent une partie des symboles et formes de la Vanité, les poétiques du XIX ${ }^{e}$ tendent à la déconstruire en opérant un déplacement axiologique du dispositif. De fait, la référence s'accompagne d'une distance critique voire d'une décomposition totale de la portée symbolique du genre. Originellement, l'objectif de la Vanité est d'inciter le lecteur ou le spectateur à mépriser les biens terrestres et de le consoler en lui faisant valoir le salut céleste. Or, nombreux sont les poètes du XIX siècle qui annihilent ce double postulat. Dans Les Fleurs du Mal, les textes que l'on peut qualifier de Vanité détournent l'argumentation par leur dimension polémique. C'est le cas de "L'Horloge " de Baudelaire dont on trouve des échos dans «Complainte des Mounis de Mont-Martre» de Laforgue :

Triturant bien l'heure en secondes,

En trois mil six cents coups de dents,

De nos parts au gâteau du temps

Ne faites qu'un hachis immonde

Devant lequel on se morfonde! (1981, p. 156) 
Dans Les Fleurs du Mal, à cette mise en scène de lécoulement du temps s'ajoutait la mention récurrente et explicite du memento mori. Dans les deux cas, toute échappatoire est refusée à l'homme :

Souviens-toi que le Temps est un joueur avide

Qui gagne sans tricher, à tout coup! c'est la loi.

Le jour décroît; la nuit augmente; Souviens-toi!

Le gouffre a toujours soif; la clepsydre se vide. (Baudelaire, 1980, p. 59)

Non que la Vanité traditionnelle soit exempte d'une certaine violence, mais le ton comminatoire est accentué par la prosopopée et l'interlocution poétique :

Tantôt sonnera l'heure où le divin Hasard,

Où l'auguste Vertu, ton épouse encor vierge,

Où le Repentir même (oh! la dernière auberge!),

Où tout te dira Meurs, vieux lâche! il est trop tard! (Baudelaire, 1980, p. 59)

Dans ce poème, le poète n'incite ni à profiter de la vie devant l'imminence de la mort, ni à se préparer à une «bonne mort » en se consolant avec l'espoir du salut céleste. Le futur pose l'avenir de l'homme comme inéluctable et irréversible. Absent, Dieu est remplacé par un symbole matérialiste du Temps, l'Horloge, « dieu sinistre, effrayant, impassible».

Si la «mort de Dieu » paraît effective, elle ne supprime pas pour autant la méditation métaphysique. "Une charogne " offre une description de la putréfaction du cadavre animal pour inciter le lecteur à réfléchir sur sa condition mais la mort y est présentée comme une étape du cycle de la vie :

Le soleil rayonnait sur cette pourriture,

Comme afin de la cuire à point,

Et de rendre au centuple à la grande Nature

Tout ce qu'ensemble elle avait joint ;

Et le ciel regardait la carcasse superbe

Comme une fleur s'épanouir. (Baudelaire, 1980, p. 23-24)

La métaphore culinaire et la référence au principe de Lavoisier témoignent de l'évolution de la pensée qui s'est largement émancipée du discours religieux. Contrairement à la fixité des Natures mortes classiques, le poème s'appuie sur une dynamique du devenir, qui préfigure les œuvres d’art modernes comme Still life (2001) de Sam Taylor Wood ou Mont Blanc (2002) et Sans titre (2002) de Michel Blazy. Ce poème est doublement provocateur. Il détourne les codes du poème sen- 
timental en rapprochant la charogne et la femme. De plus, il opère un glissement entre eros et thanatos, entre transcendance et art, inconcevable dans les Vanités classiques :

Alors, ô ma beauté ! dites à la vermine

Qui vous mangera de baisers,

Que j'ai gardé la forme et l'essence divine

De mes amours décomposés! (Baudelaire, 1980, p. 23-24)

Le poète affirme la supériorité de l'Art, par conséquent, de l'activité humaine, sur la création divine dans une sorte de démesure qui détruit totalement l'orientation initiale de l'argumentation. Pour Laforgue, qui s'adresse à la lune : "L'Art est tout, du droit divin de l'Inconscience» (1981, p. 229). Les discours tenus par les poètes vident la Vanité de sa substance théologico-morale en insistant sur la prédominance de l'art. La provocation atteint son paroxysme dans Une saison en enfer :

« Rien n'est vanité ; à la science, et en avant ! » crie l'Ecclésiaste moderne, c'est-à-dire Tout le monde. Et pourtant les cadavres des méchants et des fainéants tombent sur le cœur des autres... Ah ! vite, vite un peu ; là-bas, par delà la nuit, ces récompenses futures, éternelles... les échapperons-nous ?... (Rimbaud, 1999, p. 438)

Rimbaud inverse totalement l'argumentation originelle : point de salut possible sur terre comme au ciel. La déconstruction de la Vanité s'exerce dans le fond comme dans la forme : le poème en prose, le brouillage énonciatif font jaillir un cri de révolte qui s'oppose à la résignation imposée auparavant. Dans «L'Angoisse ", Verlaine semble inviter au mépris des divertissements humains mais c'est mieux anéantir la configuration discursive propre à la Vanité :

Je ris de l'Art, je ris de l'Homme aussi, des chants,

Des vers, des temples grecs et des tours en spirales

Qu'étirent dans le ciel vide les cathédrales,

Et je vois du même œil les bons et les méchants. (Verlaine, 1962, p. 64-65)

Le poète affiche le caractère dérisoire des activités terrestres. Cependant, en valorisant la subjectivité, il occulte l'universalité du propos. Outre le caractère personnel de l'énonciation, il annule totalement le discours d'humilité habituellement tenu dans un nihilisme généralisé :

Je ne crois pas en Dieu, j'abjure et je renie

Toute pensée, et quant à la vieille ironie,

L'Amour, je voudrais bien qu'on ne m’en parlât plus. (Verlaine, 1962, p. 64-65) 
Il place à un même niveau vie et mort, gommant le mépris de l'existence et la peur de la fin :

Lasse de vivre, ayant peur de mourir, pareille

Au brick perdu jouet du flux et du reflux,

Mon âme pour d'affreux naufrages appareille. (Verlaine, 1962, p. 64-65)

Certes, le poncif, hérité du romantisme, de l'être pris entre son dégout de la vie et sa peur de la mort se distingue de l'esthétique de la Vanité. Néanmoins, cette représentation splénétique est traitée avec une distance critique qui participe de l'évolution du genre. Le détournement passe par l'ironie et l'esthétisation de la mort, qui creuse l'écart entre représentation et signification, comme dans Les Fleurs du Mal:

L’Amour est assis sur le crâne

De l'Humanité,

Et sur ce trône le profane,

Au rire effronté,

Souffle gaîment des bulles rondes

Qui montent dans l'air,

Comme pour rejoindre les mondes

Au fond de l'éther.

Le globe lumineux et frêle

Prend un grand essor,

Crève et crache son âme grêle

Comme un songe d'or. (Baudelaire, 1980, p. 88-89)

Baudelaire reprend le symbole central de la Vanité, le crâne, ainsi que les bulles, mais le traitement allégorique et métadiscursif du thème en modifie le fonctionnement. De fait, le poète joue de l'ambigüité de ce crâne dont on ne sait s'il est anthume ou posthume :

J'entends le crâne à chaque bulle

Prier et gémir :

- « Ce jeu féroce et ridicule,

Quand doit-il finir?

Car ce que ta bouche cruelle

Éparpille en l'air,

Monstre assassin, c'est ma cervelle,

Mon sang et ma chair! (Baudelaire, 1980, p. 88-89) 
Le message est infléchi par cette équivoque qui présente non pas la peur de la mort mais la souffrance que représente la vie. Dans "Un jeune qui s'en va », ce cliché est traité de manière dérisoire par Corbière qui en fait pourtant un élément constitutif de sa réflexion :

Oh le printemps! - Je voudrais paître !...

C'est drôle, est-ce pas : Les mourants

Font toujours ouvrir leur fenêtre,

Jaloux de leur part de printemps! (2018, p. 102)

L'incorrection syntaxique s'allie à l'ironie de la métaphore animale pour montrer que l'image du poète mourant pour obtenir la gloire est dépassée. Le poète refuse l'apprentissage de la mort autant parce qu'il le considère comme une pose artificielle que parce qu'il refuse d'envisager le terme de son existence, rendue perfectible par l'écriture :

Mais non, la poésie est : vivre,

Paresser encore, et souffrir

Pour toi, maitresse ! et pour mon livre ; (Corbière, 2018, p. 102)

Si la vie est présentée comme une souffrance, elle n’en est pas moins désirable. Les poètes de la deuxième moitié du XIX ${ }^{e}$ siècle renouvellent le genre en jouant sur la proximité entre memento mori et carpe diem, souvent liés dans les représentations artistiques de la mort. Cette perméabilité transforme le genre par la conception du Temps qu'elle sous-tend. Tandis que le memento mori exhorte à mépriser le présent pour envisager sereinement l'avenir, le carpe diem incite souvent à une projection terrifiante du futur pour profiter du présent. Brouillant ces différentes catégories, les poètes annihilent l'orientation axiologique de la Vanité par la négation de la temporalité. La situation des textes dans certains recueils est éloquente : "Préludes autobiographiques » de Laforgue (1981, p. 55-59) présentent une réflexion sur la mort au début des Complaintes; «Épitaphe » constitue un des poèmes liminaires des Amours jaunes (Corbière, p. 71-75). Le déroulement chronologique et ses implications sur la vie humaine sont raillés et repoussés comme inopérants et vains comme dans « Paria»:

Mon passé : c'est ce que j'oublie.

La seule chose qui me lie

C'est ma main dans mon autre main.

Mon souvenir - Rien - C'est ma trace.

Mon présent, c'est tout ce qui passe

Mon avenir - Demain... demain. (Corbière, 2018, p. 207) 
Chaque strate temporelle est renvoyée au néant grâce aux formulations aporétiques ou tautologiques. Dès lors, le propos hérité des Vanités est tourné en dérision : sans enchaînement temporel, la peur de la putréfaction et de la mort est annihilée. Alors que le discours moral stigmatise la vanité, au sens étymologique du terme, lénonciateur revendique son aspiration au néant:

Je ne connais pas mon semblable ;

Moi, je suis ce que je me fais.

- Le Moi humain est haïssable...

- Je ne m’aime ni ne me hais. (Corbière, 2018, p. 207)

Corbière reprend explicitement le discours pascalien pour mieux l'inverser et pour tourner en dérision tout énoncé consolatoire :

- Des dieux ?... - Par hasard j'ai pu naitre ;

Peut-être en est-il - par hasard...

Ceux-là, s'ils veulent me connaître,

Me trouveront bien quelque part.

- Où que je meure : ma patrie

S’ouvrira bien, sans qu'on l'en prie,

Assez grande pour mon linceul...

Un linceul encor : pour que faire?...

Puisque ma patrie est en terre

Mon os ira bien là tout seul... (2018, p. 208)

Les motifs propres aux Vanités sont déconstruits systématiquement. La transcendance est niée ; la métonymie pour représenter le squelette dédramatise l'image effrayante de la mort. La désinvolture et l'irrévérence du discours s'allient à ce que Christian Angelet (1961) nomme la " dépoétisation concrète " que l’on retrouve également chez Laforgue, en particulier, dans la «Complainte de l'oubli des morts » :

Vous avez bien dîné,

Comment va cette affaire?

Ah ! les petits mort-nés

Ne se dorlotent guère! (1981, p. 141)

Le discours dénonce bien la vanité des préoccupations humaines et le refus de considérer la mort comme une échéance concernant les vivants mais il est tourné en dérision par les notations prosaïques : 
Notez, d'un trait égal,

Au livre de la caisse,

Entre deux frais de bal :

Entretien tombe et messe. (Laforgue, 1981, p. 141)

La portée métaphysique et morale est dégradée par les considérations matérielles. La mention du livre de compte vide de sa substance philosophique le discours des Vanités, par ailleurs discrédité par le ton léger et le rythme enlevé adoptés :

C'est gai,

Cette vie ;

Hein, ma mie,

Ô gué? (Laforgue, 1981, p. 141)

S’il existe un corpus de Vanité dans la poésie lyrique de la deuxième moitié du XIX siècle, il subit la défiguration, résultant de l'ironie inhérente à ce siècle. Iconoclaste et cynique, la poésie lyrique s'inspire de cette tradition mais pour mieux la déconstruire. Sans pour autant renoncer à sa portée métaphysique, elle la réoriente totalement. Dès lors, on peut s'interroger sur l'intérêt de reprendre la Vanité au sein d'un genre et d'une esthétique qui semblent incompatibles avec une telle tradition.

\section{La Vanité comme instrument critique du lyrisme}

En fait, dotée d'une dimension métadiscursive, la Vanité semble participer de la dynamique critique, propre au lyrisme de la deuxième moitié du XIX et contribue à le renouveler. Même si l'on assiste à une disparition progressive du sujet après l'ère romantique, il n'en reste pas moins que la poésie lyrique, miroir d'une subjectivité qui se regarde, paraît contraire au mépris du monde et à la condamnation de l'orgueil prêchés à l'Âge classique. Peut-être faudrait-il envisager le poème reprenant les codes de la Vanité comme une représentation du vide du sujet, tel que le sens étymologique le laisse penser. Mais alors, pourquoi représenter le néant si ce n'est pour lui donner une consistance et exhiber la vacuité du geste et du sujet lyriques? C'est l'une des hypothèses que l'on peut formuler en lisant « Laisser-courre » de Corbière. Suivant l'effet de liste, caractéristique des Vanités picturales, le poète reprend la tradition testamentaire héritée de Villon (Parenteau, 2006) en marquant son mépris pour les choses humaines :

J'ai laissé par les mondes,

Parmi tous les frisons

Des chauves, brunes, blondes

Et rousses... mes toisons. 
Mon épée aux vaincus,

Ma maîtresse aux cocus...

Aux portes les portières,

La portière au portier,

Le bouton aux rosières,

Les roses au rosier,

À l'huys les huissiers,

Créance aux créanciers... (Corbière, 2018, p. 153)

L'accumulation ironique des polyptotes et des calembours déconstruit de manière burlesque la logique de la Vanité. Pourtant, le questionnement existentiel est au cœur du poème :

Sous le temps, sans égides

M’a mal mené fort bien

La vie à grandes guides...

Au bout des guides - rien -

... Laissé, blasé, passé,

Rien ne m’a rien laissé... (Corbière, 2018, p. 155)

La répétition du pronom négatif en position de sujet et d’objet renvoie l'énonciateur à l'inanité de l'existence terrestre mais également d'un salut céleste. La quête d'identité parait vouée à l'échec. La Vanité dans la poésie lyrique serait donc une tentative pour peindre le néant en résolvant le paradoxe inhérent à cette tradition artistique, à savoir dire le vain en se servant d'un mode d'expression par définition chimérique. «Paria » vient corroborer cette idée :

Ma pensée est un souffle aride :

C’est l'air. L'air est à moi partout.

Et ma parole est l'écho vide

Qui ne dit rien - et c'est tout. (Corbière, 2018, p. 207)

Corbière réécrit la Vanité en déployant une écriture de la négativité qui assure au « je », synonyme de néant, une existence. L'isotopie aérienne définit la parole et le sujet lyriques comme vains mais leur donne paradoxalement une consistance poétique. Laforgue adopte une démarche similaire dans Les Complaintes. Après avoir déploré l'inanité de l'existence humaine et s'être révolté contre sa condition, il affirme la persistance de la voix lyrique :

En attendant la mort mortelle, sans mystère,

Lors quoi l'usage veut qu'on nous cache sous terre. 
Maintenant, tu n'as pas cru devoir rester coi ;

Eh bien, un cri humain! S’il en reste un pour toi. (Laforgue, 1981, p. 59)

La parole poétique ou poïétique vient faire exister le néant dans lévanescence des mots et de l'écriture. L'intertextualité et l'autoréférence font naître une œuvre autarcique qui détache l'homme de la concrétude du réel en lui donnant une existence pérenne : dire la mort pour affirmer la vie tout en sachant qu'elle ne peut être repoussée.

En réécrivant les Vanités, les poètes affirment le néant en montrant que les clichés qui constituent le genre relèvent du périssable. À l'instar de Christine BuciGlucksmann, on pourrait parler de « vanités secondes » ou de « méta-vanité » (2010, p. 54). La référence aux pratiques picturales assure la permanence du genre en mettant en abyme la construction artistique. Dans Les Amours jaunes, le premier poème de la section « Armor » s'intitule « Paysage mauvais » :

Sables de vieux os - Le flot râle

Des glas : crevant bruit sur bruit...

- Palud pâle, où la lune avale

De gros vers, pour passer la nuit. (Corbière, 2018, p. 211)

Corbière reprend le motif macabre, stigmatisant son caractère convenu et artificiel. D’ailleurs, le poème est suivi de « Nature morte » (2018, p. 212), - titre en forme de calembour -, qui met en scène un enterrement. Cette propension à sélectionner la dimension iconique de telle sorte que s'opère la déconstruction de la Vanité, recomposée dans la distance prise avec le modèle, manifeste l'intérêt pour d'autres modalités de communication. Corbière exhibe l'artifice de la construction symbolique pour mieux en dénoncer les limites. Dans « Un jeune qui s'en va », il accumule une série de portraits en raillant l'esthétisation romantique et la recherche stéréotypée de la gloire dans la mort :

Décès : Rolla : - l'Académie -

Murger, Baudelaire : - hôpital, -

Lamartine : - en perdant la vie

De sa fille, en strophes pas mal...

Doux bedeau, pleureuse en lévite,

Harmonieux tronc des moissonnés

Inventeur de la larme écrite,

Lacrymatoire d'abonnés !...

[...]

- Lord Byron, gentleman-vampire,

Hystérique du ténébreux ; 
Anglais sec, cassé par son rire,

Son noble rire de lépreux. (Corbière, 2018, p. 104-105)

Ce poème peut être qualifié de "vanité de la vanité ». Le poète figure à l'intérieur du poème cette aspiration vaine à la gloire. Il reprend les symboles traditionnels : divertissements, richesses de la "pipe/Turque, incrustée en faux saphir ", succès poétique sont tournés en dérision. Sur le plan stylistique, les formules lapidaires et la syntaxe elliptique s'inspirent des formes brèves propres à la littérature morale. Plus significative encore est la référence à la tradition du portrait médiéval et aux arts visuels. Comme l'a montré Norbert Schneider (1999, p. 77), à l'origine, on trouvait au verso des portraits des crânes rappelant la putrescibilité du corps, d’où le lien entre l'image du sujet et l'image de sa mort à venir. En l'occurrence, le traitement ironique des portraits dans le portrait infirme la portée morale du propos. La mise en abyme de la figure du poète, écho des Vanités baroques, crée un vertige de la représentation. L'image est démultipliée dans le temps et dans l'espace : la vanité lyrique devient le miroir dans lequel le sujet contemple son propre néant.

Dès lors, la représentation sémancipe de la signification pour transmettre différemment les émotions. La perception sensorielle prend le pas sur l'appréhension intellectuelle pour susciter des images, des sons qui se suffisent à eux-mêmes. De fait, plusieurs poèmes ne semblent retenir que le potentiel sensoriel de la tradition de la Vanité. Le poème "Heures », au titre révélateur, manifeste le glissement qui s'opère dans le fonctionnement de la vanité. Corbière met en scène un personnage imaginaire, moyen de mettre à distance le " je », confronté à la mort. L'atmosphère lugubre naît de la référence visuelle associée aux notations auditives :

J'entends comme un bruit de crécelle...

C'est la male heure qui m'appelle.

Dans le creux des nuits tombe : un glas... deux glas (Corbière, 2018, p. 140)

Lénonciateur constitue la médiation qui permet au lecteur de se projeter dans la représentation. La référence à l'instrument des lépreux permet d'évoquer à la fois une sonorité dysphorique et l'image de la putréfaction du corps. La versification accentue l'effet mortifère du texte. Le passage de l'octosyllabe au décasyllabe insiste sur le caractère oppressant du déroulement du temps :

J'ai compté plus de quatorze heures...

L'heure est une larme - Tu pleures,

Mon cœur !... Chante encor, va - Ne compte pas. (Corbière, 2018, p. 140)

La fin du poème suggère une prolongation infinie du temps et du chant lyrique, affirmant sa permanence tout en disant la précarité du sujet. De fait, la mascarade fait partie des effets spéculaires qui font de la Vanité un miroir du « je » et de sa labilité. 
On assiste à la fin du régime symbolique pour entrer dans l'ère de l'expérience vécue, le temps de la lecture par la coexistence de plusieurs « objets mentaux » (Bonnefoy, 1999, p. 214). Dans «Une charogne ", le lecteur ressent physiquement l'expérience vécue par le sujet lyrique, en lui faisant éprouver les effets de la putréfaction :

Les mouches bourdonnaient sur ce ventre putride,

D'où sortaient de noirs bataillons

De larves, qui coulaient comme un épais liquide

Le long de ces vivants haillons. (Baudelaire, 1980, p. 23-24)

Baudelaire mobilise tous les sens pour transmettre au récepteur ce spectacle. Le lecteur n'est plus mis en garde directement par un système de codification symbolique mais par l'évocation sonore, olfactive, tactile et visuelle de la représentation. Il ressent les sensations et les émotions suscitées par les objets mentaux que font naître les mots. Dès lors, il doit prendre en charge le discours latent dans la sphère où se rencontrent systèmes visuel et sonore. En témoigne la "Complainte des Complaintes » :

Maintenant! pourquoi ces complaintes?

Gerbes d'ailleurs d'un défunt moi

Où l'ivraie art mange la foi ? [...]

Chut ! Tout est bien, rien ne s’étonne.

Fleuris, ô terre d'occasion,

Vers les mirages des sions!

Et nous, sous l'art qui nous tâtonne,

Sisyphes par persuasion,

Flûtant des christs les vaines fables,

Au cabestan de l'incurable

POURQUOI ! - Pourquoi ? (Laforgue, 1981, p. 169)

Le questionnement est suspendu à la contribution du lecteur qui doit participer à la construction symbolique. La néantisation du sujet rejaillit sur l'interlocuteur qui prend à son tour en charge la vanité de l'existence. Grâce à la distance critique prise avec le modèle, les poètes ménagent un espace vacant au sein duquel l'angoisse face à la mort, indicible, se transmet sans discours moral mais grâce à l'expérience émotionnelle et perceptuelle qu'en fait le lecteur.

La transposition littéraire des codes picturaux à une époque d'instabilité et de bouleversements tels que les a connus le XIX ${ }^{e}$ siècle génère un infléchissement du fonctionnement symbolique et formel de la Vanité. Cependant, la reprise d'un certain nombre d'invariants déjà présents à l'époque classique permet de constituer un corpus de vanités dans la poésie lyrique de Baudelaire à Laforgue : le sujet se livre à une réflexion sur sa condition et cherche à transmettre son désarroi. Le contexte théologico-moral s'est dissous pour laisser place à une réflexion, dans les deux sens du terme : 
pensée sur l'existence et miroir de cette pensée. La Vanité devient un moyen de mettre en scène la vacuité du sujet et la vanité de sa représentation dans la poésie lyrique en faisant circuler les émotions différemment.

\section{RÉFÉRENCES}

Angelet, C. (1961). La Poétique de Tristan Corbière. Bruxelles, Belgique : Palais des Académies. Baudelaire, Ch. (1980). Les Fleurs du Mal [1857]. Euvres complètes. Paris, France : Laffont.

Bonnefoy, Y. (1999). Lieux et destins de l'image. Un cours de poétique au Collège de France. Paris, France : Seuil.

Buci-Glucksmann, C. (2010). Les vanités secondes, Dans Charbonneaux A.-M. (dir.), Les Vanités dans l'art contemporain (p. 54-84). Paris, France : Flammarion.

Chassignet, J.-B. (1967). Le Mespris de la vie et consolation contre la mort [1594]. H.-J. Lope (éd.). Genève, Suisse : Droz.

Corbière, T. (2018). Les Amours jaunes [1873]. Éd. J.-P. Bertrand. Paris, France : GF Flammarion. Gleizes, D. (2002/2004). Vanités : codes picturaux et signes textuels. Romantisme, 118. 75-91. Hugo, V. (1972). Les Contemplations [1856]. Paris, France : Librairie générale.

Laforgue, J. (1981). Les Complaintes et L'Imitation de Notre-Dame La lune [1885]. Paris, France : Imprimerie nationale.

Keller-Rahbé, E. (2005). Vanité et création romanesque dans la seconde moitié du XVIII siècle. Littératures classiques, 56(1). 245-266. Récupéré de https://www.cairn.info/revue-litteratures-classiques1-2005-1-page-245.htm

Lanini, K. (2006). Dire la vanité à l'âge classique. Paris, France : Champion.

Parenteau, O. (2006). Tristan Corbière, lecteur de François Villon. Études littéraires, 372. 113131.

Rimbaud, A. (1999). Une saison en enfer [1873]. Euvres complètes. Paris, France : Librairie générale française.

Rollinat, M. (1885). Les névroses : les âmes, les luxures, les refuges, les spectres, les ténèbres. Paris, France : Charpentier. Récupéré de https:/gallica.bnf.fr/ark:/12148/bpt6k107923q/f6.item

Schneider, N. (1999). Les Natures mortes. Cologne, Allemagne : Benedikt Taschen.

Verlaine, P. (1962). Poèmes saturniens [1866]. Euvres poétiques complètes. Éd. Jacques Borel. Paris, France : Gallimard.

RÉSUMÉ : À son apogée au XVII' siècle, la Vanité, grâce à sa codification, perdure au-delà de cette époque propice à son épanouissement. De même que la dimension religieuse et dogmatique tend à s'estomper au XIX', les symboles de la vacuité et de l'illusion existentielle sont repris de manière partielle. Peut-on alors encore parler de Vanité quand le discours symbolique est lacunaire ou incomplet ? De Baudelaire à Laforgue, les poétiques du XIX ${ }^{e}$ siècle opèrent un déplacement axiologique qui tend à annihiler la portée argumentative initiale. Dès lors, pourquoi reprendre une tradition apparemment incompatible avec le discours lyrique ? En fait, dotée d'une dimension métadiscursive, la Vanité semble participer de la dynamique critique, propre au ly- 
risme de la deuxième moitié du XIX ${ }^{e}$ siècle et contribue à le renouveler. Entre sérieux et ironie, la transposition picturale de la Vanité favorise un dialogue intersémiotique qui ménage un espace vacant pour un autre mode de transmission des sentiments.

Mots-clés : lyrisme critique, métadiscours, déconstruction

\section{Lyricism of the vanity and vanity of the lyricism.} A few examples of poetics of the 19th century, from Baudelaire to Laforgue ABSTRACT: At its peak in the 17th century, Vanity, thanks to its codification, persists beyond that age favourable to its blossoming. Just as the religious and dogmatic dimension tends to fade in the 19th century, the symbols of vacuity and existential delusion are partly used again. Can we still talk about Vanity then, when the symbolic discourse is incomplete? From Baudelaire to Laforgue, the poetics of the 19th century bring about an axiological transfer which tends to annihilate the initial argumentative impact. Consequently, why revive a tradition which is apparently incompatible with the lyrical discourse? In fact, endowed with a metadiscursive dimension, Vanity seems to relate to the critical dynamic specific to the lyricism of the second half of the 19th century and contributes to renew it. Between seriousness and irony, the pictorial transposition of Vanity fosters an intersemiotic dialogue which makes room for another method of transmission of feelings.

Keywords: critical lyricism, metadiscursive, deconstructing 